\title{
Fluorescence Recordings of Electrical Activity in Goldfish Optic Tectum in vitro
}

\author{
Paul B. Manis a and John A. Freeman \\ Department of Cell Biology, Vanderbilt University School of Medicine, Nashville, Tennessee 37232
}

\begin{abstract}
Optical methods for recording electrical activity in the goldfish optic tectum were evaluated. Tectal slices, with a short section of the optic nerve attached, were stained with a fluorescent styryl dye. Potential-dependent fluorescence changes following optic nerve stimulation were monitored with a photodiode. We found that large optical signals could be obtained. Experimental manipulations of the slice bathing solution permitted us to identify several events that contributed to the optical response, including activity in afferent fibers, excitatory and inhibitory postsynaptic potentials, and presumptive glial depolarizations. These results suggest that voltage-sensitive dyes can provide a useful alternative method for monitoring synaptic responses in the goldfish tectum, and may prove valuable in studying changes in the functional synaptic organization of the tectum following manipulations of the retinotectal pathway.
\end{abstract}

The goldfish retinotectal system has long served as a model system for the study of nerve regeneration and the factors controlling topographic organization of synaptic connections. Physiological studies on the organization of retinotectal connections have relied on the results of single and multiunit extracellular recordings. The origin of the unitary events observed in these studies has been ascribed almost universally to activity in presynaptic arbors. However, it is possible that the functional synaptic connectivity may differ significantly from the afferent terminal distribution. The functional connectivity is best determined by measuring postsynaptic events. Postsynaptic responses have been studied during development by Chung et al. (1974) and following manipulations of the retinotectal system by Freeman (1977), Boss and Schmidt (1984), and Schmidt et al. (1983). In all of these studies, current source-density (CSD) analysis of extracellularly recorded field potentials was used to assess the functional state of synaptic transmission as a result of shocking the optic nerve. Only one study (Freeman, 1977) actually evaluated the spatial distribution of responses to visual stimulation. However, retinotectal mapping using CSD analysis

\footnotetext{
Received Nov. 4, 1985; revised July 24, 1987; accepted July 29, 1987.

We would like to thank Dr. Amiram Grinvald for his generous gift of the voltagesensitive dyes and his comments on the manuscript, and Ms. Susan Bock and Mr. Philip Samson for their able technical assistance with these studies. This work was supported by a National Eye Institute Grant EY 01117 to J.A.F. and a National Research Service Award NS07377 to P.B.M.

Correspondence should be addressed to Paul B. Manis, Department of Otolaryngology-Head and Neck Surgery, The Johns Hopkins University School of Medicine, 426 Traylor Research Bldg., 720 Rutland Avenuc, Baltimore, MD 21205.

- Present address: Departments of Otolaryngology-Head and Neck Surgery, and Neuroscience, The Johns Hopkins University School of Medicine, 426 Traylor Research Bldg., 720 Rutland Avenue, Baltimore, MD 21205.

Copyright (C) 1988 Society for Neuroscience $0270-6474 / 88 / 020383-12 \$ 02.00 / 0$
}

is difficult because of the need for multiple electrode penetrations and long-term physiological stability of the preparation. Similarly, intracellular recordings in the tectum are difficult, particularly because of the small size of the cells. A more rapid and direct approach for mapping postsynaptic responses over a large area would be useful.

Optical recording using voltage-sensitive dyes (see Cohen and Salzberg, 1978; Grinvald and Segal, 1984; Grinvald, 1985) offers the possibility of using a photodiode array to perform mapping studies of postsynaptic responses rapidly and relatively noninvasively. These dyes can signal membrane potential changes of even the smallest neural or glial elements. The optical signals arise from either changes in dye transmission at a particular wavelength or from changes in the dye fluorescence when excited at an appropriate wavelength. The optical changes are linearly related to membrane potential and have been shown to occur extremely rapidly following changes in potential (Cohen et al., 1974; Ross et al., 1977; Gupta et al., 1981; Loew et al., 1985). Absorption dyes were used in a previous study to examine potential changes in the hippocampal slice preparation (Grinvald et al., 1982b). That study showed that optical signals with a high signal-to-noise ratio could be obtained from dendritic regions and unmyelinated fibers and that the optical changes had a clear relationship to the known neurophysiology. Mapping studies in the frog tectum (Grinvald et al., 1984) showed that the dye responses accurately reflected visuotopic organization.

As the first step in implementing these techniques, we have evaluated the use of optical recording methods for the study of postsynaptic responses in the goldfish retinotectal system and have performed experiments to determine the cellular and synaptic origins of portions of the optical signal. Specifically, we have examined the fluorescence signal of certain styryl dyes in goldfish optic tectum slices in vitro. Since the majority of neurons in the goldfish optic tectum are of one class (type XIV, as described by Meek, 1983), and since these neurons have radial dendrites extending from the cell bodies in stratum periventriculare (SPV) to stratum opticum (SO), we expected that the neural contribution to optical signals recorded in these layers following optic nerve activation would be associated primarily with membrane potential changes in these cells. However, other neuronal cell types may also contribute. The goldfish tectum also has a large population of radial glial cells, whose processes extend from the pial to the ventricular surface (Stevenson and Yoon, 1982). A non-neuronal membrane potential component in all laycrs might be expected from these cells. We have identified multiple components in the optical response, and present preliminary evidence concerning their origin. These components include the activity in afferent fibers and possibly their en- 
sheathing glial cells, a mono- or disynaptic EPSP, an IPSP, a late EPSP, an EPSP apparently arising from disinhibition in the presence of convulsant agents, and a probable glial depolarization associated with the postsynaptic potentials. In addition, we have observed a pharmacological effect of the dyes when applied to the tectum in relatively high concentrations. Portions of this work have been presented previously in abstract form (Manis and Freeman, 1984, 1985).

\section{Materials and Methods}

Preparation. An in vitro slice preparation of the goldfish optic tectum (Freeman, 1979a, b; Matsumoto et al., 1983) was employed. Goldfish were anesthetized by immersion in ice water. When all movement ceased, the fish were placed on the stage of a dissecting microscope and the cranial cavity fully exposed. The brain was transected between the medulla and spinal cord, and while gently lifting with a spatula from the caudal end, the cranial nerves were cut with fine scissors to free the brain. The brain was placed in ice-cold HEPES buffered goldfish Ringer's (see below for composition), and the midbrain dissected free of the telencephalon and cerebellum. The midbrain, including the tectum, was placed on Ringer's-soaked filter paper on the stage of a tissue chopper. The tectum was tilted so that the medial margin was parallel to the plane of the razor blade. Thin $(200-250 \mu \mathrm{m})$ slices of the lateral optic tectum were cut, and one thick $(500 \mu \mathrm{m})$ slice was cut at the medial aspect of the tectum. Properly cut thick slices included a short section of both the dorsal and ventral branches of the optic tract. These slices remain physiologically viable as assessed by the appearance of extracellular synaptically generated field potentials of $2-4 \mathrm{mV}$ in amplitude consequent to optic tract stimulation for at least 8 (and up to 24) $\mathrm{hr}$ under normal conditions. The thin lateral slices rarely showed extracellular field potentials following stimulation at the rostral end, probably because the trajectory of the optic nerve fibers that innervate the central tectum is curved (Attardi and Sperry, 1963; Cook et al., 1983; Rusoff, 1984; Stuermer, 1984), and these fibers would have been sectioned before reaching terminal destinations. Only at the medial margin of the tectum are both the optic nerve fibers and their terminals included in a thin parasagittal slice.

The slices were incubated in a goldfish Ringer's at room temperature $\left(18-20^{\circ} \mathrm{C}\right)$ for at least $1 \mathrm{hr}$ before physiological experiments. The incubation and standard recording media contained $112 \mathrm{~mm} \mathrm{NaCl}, 3 \mathrm{~mm}$ $\mathrm{KCl}, 2.5 \mathrm{~mm} \mathrm{CaCl}_{2}, 0.5 \mathrm{~mm} \mathrm{MgCl}_{2}, 0.5 \mathrm{~mm}, \mathrm{MgSO}_{4}, 1 \mathrm{~mm}$ Na-Pyruvate, $16 \mathrm{~mm}$ glucose, $0.5 \mathrm{~mm} \mathrm{NaH} \mathrm{PO}_{2}$, and $20 \mathrm{~mm} \mathrm{NaHCO}$. The pH was $7.35-7.45$ when equilibrated with $95 \% \mathrm{O}_{2}-5 \% \mathrm{CO}_{2}$. The dissection medium was similar to the recording media, except that it contained 122 $\mathrm{mm} \mathrm{NaCl}, 10 \mathrm{~mm}$ HEPES, and no $\mathrm{NaHCO}_{3}$. The dissection medium was air-equilibrated, and the $\mathrm{pH}$ was adjusted to 7.4 with $\mathrm{NaOH}$. Ionic substitutions were performed on an iso-osmolar basis. (In particular, when the potassium concentration was altered, the sodium levels were adjusted to maintain osmolarity.) When used, bicuculline methiodide, strychnine, and $d$-tubocurarine were added to the standard medium immediately before the experiment. All experiments were performed at room temperature $\left(18-20^{\circ} \mathrm{C}\right)$.

After the $1 \mathrm{hr}$ incubation, a slice was transferred to the recording chamber on the microscope stage (see Optical recordings). The chamber was made of Plexiglas, with a glass coverslip bottom for visualization of the slice with high-magnification optics. The slice was held gently in place with a small piece of netting (bridal veil) glued to a rubber O-ring. The total chamber volume, including the side port for the reference electrode, was about $0.24 \mathrm{ml}$; the effective exchange volume with the slice and O-ring in place was about $0.1 \mathrm{ml}$. Recording and stimulating electrode micromanipulators were mounted on the microscope stage. The stage could then be moved to visualize different regions of the slice while maintaining fixed the relationship between the electrodes and the slice.

The optic tract was stimulated with a bipolar electrode using $0.1 \mathrm{msec}$ square pulses supplied by an optically isolated constant-voltage stimulator. Supramaximal stimuli (as judged from the extracellularly recorded field potentials) were used. Field potentials were recorded with Ringer's-filled glass micropipettes, beveled to $20-50 \mathrm{M} \Omega$ with a jet stream beveller. A low-noise, custom capacity-compensated amplifier was used for electrical recordings. The stimulus artifact was removed from the electrical recordings with a stimulus-artifact suppression circuit follow- ing the recording amplifier (Freeman, 1971). Optical and electrical responses were signal-averaged (usually 4-16 repetitions). Data were collected using 2 channels of a 10-bit A-D converter at $5 \mathrm{kHz}$ per channel sample rate. Electrical and optical signals were amplified and low-pass filtered at 1 or $3 \mathrm{kHz}$ with Tektronix $26 \mathrm{~A} 2$ and $7 \mathrm{~A} 12$ amplifiers, respectively.

Optical recordings. Optical recordings of the small voltage-dependent fluorescence changes of voltage-sensitive dyes were made using both an upright Olympus VANOX and an inverted Zeiss IM-35 microscope. (The recordings shown in this paper were all obtained using the inverted microscope.) Our optical recording system is nearly identical to that described by Grinvald et al. (1983) for optimal recording of single cells in tissue culture. Both microscopes were equipped with a Zeiss FL-2 epi-illumination system and dichroic mirror FT-580. A $100 \mathrm{~W}$ halogentungsten lamp (excitation filter, $540 \mathrm{~nm} ; 30 \mathrm{~nm}$ half-width) or a $100 \mathrm{~W}$ HBO mercury-arc lamp (excitation filter, $546.1 \mathrm{~nm} ; 12.7 \mathrm{~nm}$ half-width) were used to excite dye fluorescence. An OG-580 barrier filter was used to separate excitation light from the dye fluorescence. The data shown were collected with $40 \times 0.60 \mathrm{NA}$ LWD and $40 \times 0.75 \mathrm{NA}$ Neofluar Zeiss objectives.

The fluorescence was detected with a PV-100 (EG\&G) photodiode mounted on the camera adapter (Olympus VANOX) or TV-cine adapter (Zeiss IM-35). An opaque mask with a small centrally located pinhole was inserted in the camera reticle carrier to limit detected fluorescence to a $50-\mu \mathrm{m}$-diameter spot in the object plane. In some experiments, a rectangular $100 \times 120 \mu \mathrm{m}$ aperture was used. A low-noise I-V converter $\left(R_{\mathrm{r}}=22,200\right.$, or $\left.750 \mathrm{M} \Omega\right)$ provided the initial amplification of the optical signals. Exposure of the preparation to the intense illumination necessary to obtain a good signal-to-noise ratio was minimized by using a shutter (Vincent Assoc., Rochester, NY). A second photodiode in the transmitted light path provided a reference signal for an analog automatic gain-tracking reference subtraction circuit, which eliminated lamp noise from the optical traces in real time (Manis et al., unpublished observations). The slices transmitted between 25 and $50 \%$ of the incident light. A calibration pulse was included on the optical recordings by adding a fraction of the resting fluorescence signal to the recording with a gated summing circuit.

We optimized our recording system for detection of small fluorescence changes from the slices in 2 ways. First, for most of the experiments, the aperture of the luminous field diaphragm on the microscope was reduced so that an area of the slice only slightly larger than that viewed through the pinhole aperture was illuminated. This had the desirable consequence of minimizing the scattered fluorescence from adjacent regions of the slice (because they were not illuminated), and thus permitting a finer spatial localization of responses than would be obtained by using the full illumination aperture. Reducing the luminous aperture also reduces the effective numerical aperture of the illumination system to a value significantly below that provided by the objective used (i.e., the total optical flux of the illumination system has been reduced; see Piller, 1977). Thus, the detected fluorescence level was low. However, by using the highest gain on the I-V converter, a $1 \mathrm{kHz}$ bandwidth, and the mercury-arc lamp, it was possible to obtain recordings where the light shot noise exceeded the dark noise of the photodiode-amplifier combination by a factor of 4 . The detected resting fluorescence in these cases was measured at $7 \times 10^{10}$ photons $/ \mathrm{sec}$, implying a potential signalto-noise $(\mathrm{S} / \mathrm{N})$ ratio of about $50: 1 \mathrm{rms}$ for a $1 \%$ change in fluorescence. Very little bleaching was observed under these conditions.

Second, for some experiments we sought to reduce our noise floor enough to be able to observe the even smaller fluorescence changes $(<0.1 \%)$ associated with activity in the afferent fibers. In these cases, we used the larger rectangular aperture, and illuminated a larger region of the slice. This resulted in approximately 35 times more detected fluorescence and a correspondingly lower shot noise (see Fig. 8D). However, significant bleaching was observed under these conditions, and it was necessary to correct the time course of the traces. A bleaching correction similar to that described by Grinvald et al. (1982a) was applied. Control bleaching records were taken by recording the optical signals from the slices without stimulation. These records were taken immediately after the records taken with stimulation to insure identical conditions. The resting fluorescence level computed from the calibration pulse at the beginning of the optical traces was added back to the data before the bleaching correction. The control bleaching records were fit to a third-order polynomial, which was then divided into the experimental records. The resulting corrected experimental trace was then scaled back to the original size using the calibration pulse for reference. 
The validity of the bleaching correction was verified by noting that the time courses of records taken at different bleaching levels were nearly identical after this correction.

The voltage-sensitive dyes used in this study were the generous gift of Dr. Amiram Grinvald (Rockefeller University, NY). Some of these dyes have recently become commercially available through Molecular Probes, Inc. (Junction City, OR), and we have used these dyes with similar results. (Figures 1 and 8 were obtained with RH414 from Molecular Probes, while the rest of the figures were obtained using Grinvald's dyes.) Dyes were applied topically to the slice at $0.05-1 \mathrm{~mm}$ concentration in HEPES-buffercd Ringer's, with the perfusion system off. The staining period lasted $15 \mathrm{~min}$, after which the perfusion was reinstated. A 10 min period ensued before optical recordings commenced. Electrical records are shown with negative up, and calibration pulses are $1 \mathrm{mV}$ by $2 \mathrm{msec}$. Optical records are shown with decreasing fluorescence up. (With these styryl dyes, decreasing fluorescence corresponds to intracellular depolarization; see Gupta et al., 1981; Grinvald et al., 1982a, 1983, 1984; Orbach and Cohen, 1983; Loew et al., 1985; Orbach et al., 1985.) The amplitude of the calibration pulse on the optical traces is noted in the figure legends.

\section{Results}

\section{Extracellular field potentials recorded in tectal slices}

The extracellular field potential recorded in the SFGS of the tectal slice (Fig. 1, trace A) consists of several components. These include a presynaptic deflection (N1), a presumed monosynaptic current sink (N2, N3), a positive wave (P1), a late brief negative wave (N4), and a slow negative wave (N5). The N1 corresponds to waves 2 of Vanegas et al. (1971) and Ml of Schmidt (1979). $\mathrm{N} 2$ and N3 correspond to waves 4 and 5 of Vanegas et al. (1971), and waves P1 and P2 of Schmidt (1979). The latency of the P1 wave is similar to that of wave 6 of Vanegas et al. (1971) and to wave P3 of Schmidt. The presynaptic deflections 1 and 3 of Vanegas et al. (1971) or E, M2, and M3 of Schmidt (1979) were not consistently identifiable in our slice preparation. The N4 and $\mathrm{N} 5$ waves have been described in other reports on in vitro tectal preparations (Matsumoto and Bando, 1981; Teyler et al., 1981) but are not often seen in vivo (Vanegas et al., 1971; Schmidt, 1979; however, see Konishi, 1960). Since our recordings were done at room temperature, they have a faster time course than those shown by Schmidt (1979), which were obtained from fish cooled to $11^{\circ} \mathrm{C}$. Also, because of the proximity of the stimulation electrode to the recording site, there is probably a greater overlap between response components mediated by the different conduction velocity afferents (Schmidt, 1979) than in vivo. Nevertheless, the field potentials in the slice exhibit the same changes in form and inversion with electrode depth (pia to ventricle) as reported in vivo in several species of fish.

The N4 wave is particularly sensitive to a variety of manipulations of the extracellular milieu. It is reversibly blocked by $0.2 \mathrm{~mm}$ tricaine methanesulfonate (TMS, a commonly used fish anesthetic), $1 \mathrm{mM}$ DL- $\alpha$-aminoadipate, variations in the extracellular calcium and potassium concentrations, changes in the bath $\mathrm{pI}$, and the addition of various convulsant agents to the bath. As described below, it is irreversibly blocked by staining the slice with millimolar concentrations of voltage-sensitive dyes. We have observed this potential in slices maintained $24 \mathrm{hr}$ following initial slicing of the tectum. While this potential might represent the action of unmyelinated or myelinating fibers growing into the tectal margin as suggested by Teyler et al. (1981), its differential pharmacological sensitivity relative to the N2 and N3 responses suggest that has a different origin. One likely possibility is that it represents polysynaptic events consequent to optic nerve activation. The observation that the $\mathrm{N} 4$ potential can be observed in vivo using intraorbital stimulation of the
Field Potentials
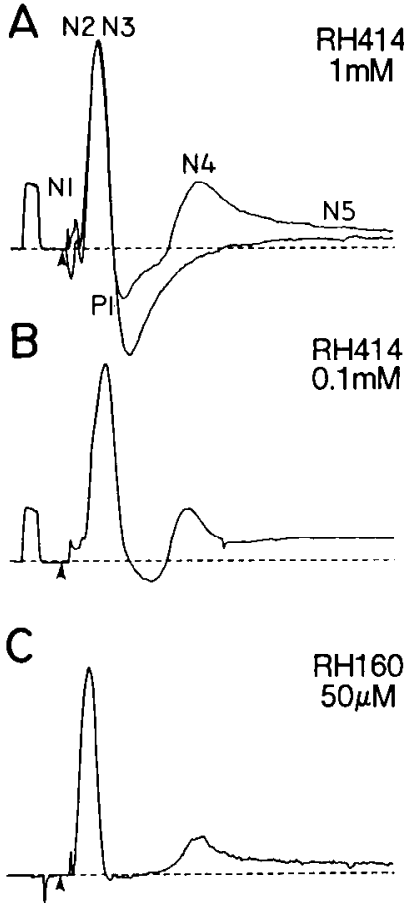

RH160

$50 \mu \mathrm{M}$

Figure 1. Field potentials and simultaneously recorded optical signals. The components of the extracellular field potential recorded in the tectal slice are labeled in the trace in $A$. Staining the slice with $1 \mathrm{~mm} \mathrm{RH414}$ altered the tectal field potential significantly $(A)$. However, large optical changes with good signal-to-noise ratios were obtained $(D)$. The 3 major components of the optical signal are labeled in $D$. At lower staining concentrations $(100 \mu \mathrm{M}, B)$, there was little effect on the field potential, but the signal-to-noise ratio was degraded because of the lowered fluorescence level $(E) . C$ and $F$, Electrical and optical responses obtained when the slice was stained with $50 \mu \mathrm{M}$ RH160. Note the suggestion of a small late depolarizing event associated with the late field potential component (N4 wave) as marked by the dot above the trace in $F$. Optical calibrations: traces $D$ and $E, 2 \times 10^{-3} \Delta F / F$ (fractional change from the resting fluorescence level); trace $F, 5 \times 10^{-3} \Delta F / F$, duration $2 \mathrm{msec}$. Decreasing fluorescence (corresponding to depolarization) is shown upwards in all optical recordings in this paper.

optic nerve head (Konishi, 1960) suggests that it is not likely to be produced by local stimulus current spread or stimulation of fibers of passage of nonretinal origin in the optic tract.

\section{Dye staining and optical signals}

The effect of topical application of the voltage-sensitive dye RH414 at a concentration of $1 \mathrm{~mm}$ is shown in Figure $1 A$. The presumed monosynaptic responses $(\mathrm{N} 2, \mathrm{~N} 3)$ are not affected by the dyc, whilc the positive wave (P1) appears to be enhanced. Whether this indicates that a portion of the P1 potential was masked by a superimposed negative potential or represents a true enhancement cannot be determined from these records. A gradual and irreversible abolition of the N4 wave is consistently observed when the slices are stained with this relatively high concentration of the dye.

Following dye staining, large optical signals with excellent signal-to-noise ratios could be recorded (Fig. $1 D$ ). No stimulusdependent optical signals were obtained in the absence of dye staining. Light scattering signals were not investigated; however the stimulus-dependent fluorescence signals that we record are 

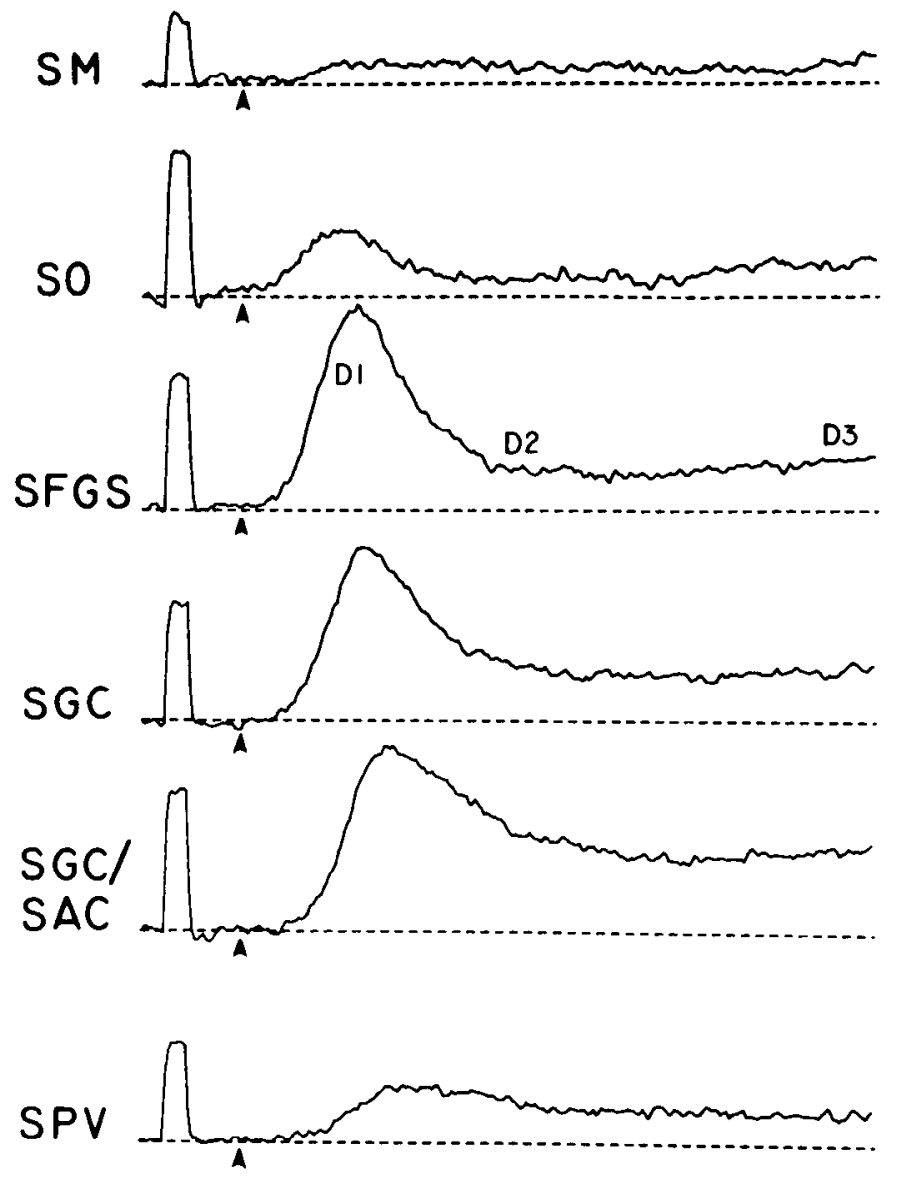

Figure 2. Laminar comparison of optical signals. Each layer was successively imaged onto the photodiode through a $50-\mu \mathrm{m}$-diameter (in the object plane) circular pinhole. The stimulus was delivered at the time indicated by the arrow beneath the traces. All traces are shown to the same scale, so the height of the calibration pulse indicates the relative resting fluorescence level. The duration of all traces is $51.2 \mathrm{msec}$. Calibration pulses are $5 \times 10^{-3} \Delta F / F, 2 \mathrm{msec}$ in duration. Dye concentration in this and all following figures is approximately $0.5 \mathrm{~mm}$. The records are the average of 4 responses.

1-2 orders of magnitude larger than the light scattering signals previously reported (Cohen ot al., 1972; Grinvald et al., 1982b). Thus, our fluorescence measurements are not likely to be contaminated by light scattering signals.

The middle row of Figure 1 (traces B and E) illustrates the response obtained after another slice was stained with $100 \mu \mathrm{M}$ RH414. The N4 wave was not abolished when this lower concentration of dye was used. The optical signals obtained from this slice are somewhat similar in form, although the fractional fluorescence change is smaller, and there appears to be a small signal associated with the $\mathrm{N} 4$ wave. The bottom row (traces $\mathrm{C}$ and $F$ ) shows responses obtained in the presence of the zwitterionic dye RH160 at $50 \mu \mathrm{M}$. The optical trace is similar in shape to that obtained with RH414. The N4 wave is still present, as is a small optical signal occurring at that time (dot above trace F). Optical signals with similar time courses were also obtained with RH421, RH355, and RH246.

The largest signals we have recorded to date are $1.5 \times 10^{-2}$ $\Delta F / F$. These signals were obtained using the dye $\mathrm{RH} 414$ and a pinhole to limit the optical signal to that coming from SFGS. Typical signals are $5-7 \times 10^{-3} \Delta F / F$, corresponding to approximately a 30:1 rms signal-to-noise ratio using the $50 \mu \mathrm{m}$ pinhole.
Occasionally the optical signals were very small or undetectable. RH160 gave signals ranging from $3-7 \times 10^{-3} \Delta F / F$, while the other dyes all produced signals at $3-5 \times 10^{-3} \Delta F / F$. The rest of the optical recordings presented in this paper were obtained with the photodiode aperture aligned over SFGS, using the dye RH414 at a concentration of $0.2-0.5 \mathrm{~mm}$.

The optical signal had a stereotyped form under normal conditions. For reference, we have labeled the largest components in Figure $1 D$. The optical responses consisted of an early fluorescence decrease (depolarization) approximately $10 \mathrm{msec}$ in duration (labeled D1), followed by a long-duration depolarization lasting at least $1 \mathrm{sec}$ (D3). In many cases, a period of lesser depolarization (or relative hyperpolarization) intervened approximately during the time labeled D2. A small inflection often appeared on the onset of the initial depolarization. Under normal conditions, all optical changes were in the direction of decreasing fluorescence.

\section{Laminar distribution of the optical signal}

In order to investigate the origins of the various components of the optical response, we examined their spatial distribution within the tectal slice. Fluorescence signals observed in different tectal layers following shock of the optic tract are compared in Figure 2. These recordings were obtained by successively moving the recording stage of the microscope, while using the pinhole to limit the optical signal to a $50-\mu \mathrm{m}$-diameter spot over a given tectal layer (as described in Methods and Materials), at a constant distance from the stimulating electrode. As can be seen, the shape of the optical signal varies among the layers. The largest and shortest latency component visible in these records is D1. The D1 signal was recorded from layers SO through SPV, but not SM. Although not pronounced in these records, D1 is followed by a period of decreased fluorescence, labeled D2. The D2 signal appeared to be maximal in SFGS and SGC. In contrast to the D1 signal, D3 appears to be present in all layers. The optical signal in stratum fibrosum marginale (SM; top trace of Fig. 2) suggests that D3 may actually begin simultaneously with D1. The onset is masked by D1 in the other layers. Although we have not been able to determine the exact duration of D3 due to low-frequency vibration noise, it appears to last at least 1 sec. The D1 optical signal appears to be preceded by a small step-like event in layers SO through SAC (see also Figs. 3 and 8).

The amplitude of the optical signal cannot readily be compared between different layers because the factors that determine the signal size are likely to vary between layers (see Grinvald, 1985). However, the time courses of the fluorescence changes can be compared. The shortest latency of the early (D1) response is found in SO and SFGS. This would be expected, as many of the optic fibers arrive through SO and synapse in these layers. The longer latency of this signal in SFGS, SGC, and stratum album centrale (SAC) suggests conduction towards SPV. The conduction velocity of this depolarization estimated from either the onset latency or the peak latency is about $0.05 \mathrm{~m} / \mathrm{sec}$, a value commensurate with passive propagation in the thin radial dendritic arbors or in unmyelinated fibers or terminal branches. Interestingly, the rise time of the D1 (measured from the foot of the signal to the peak) remains nearly constant from SFGS to SAC. This could indicate the presence of an active membrane conductance activated by the synaptic input in neural elements in these layers or could result from slightly delayed synaptic inputs to more than one region of the dendritic tree. A candidate 

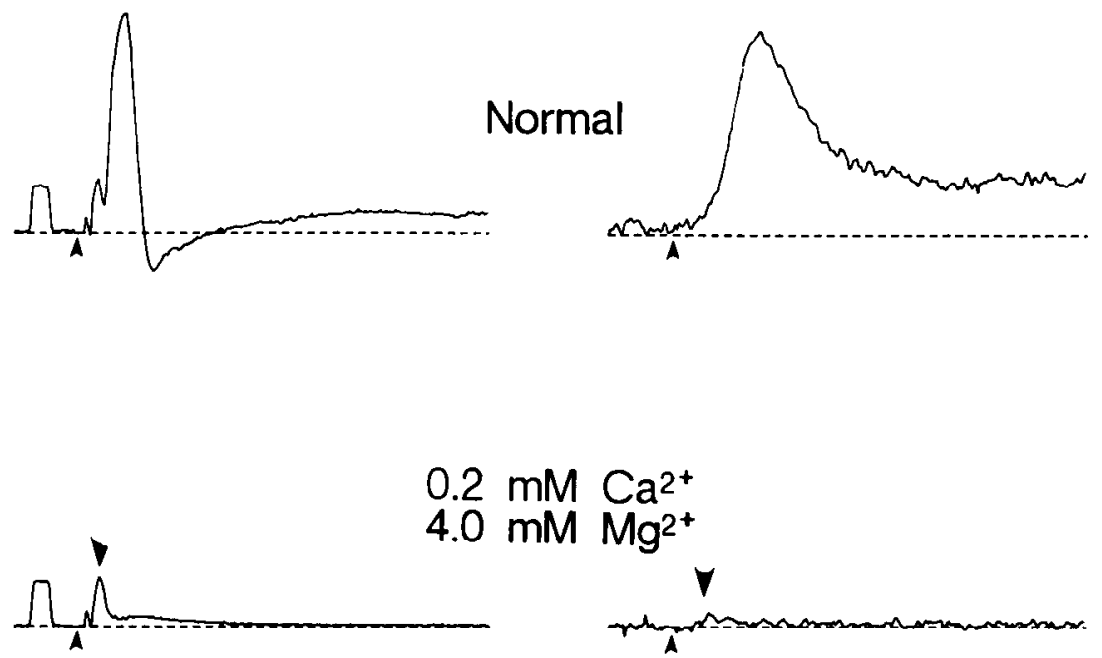

$0.2 \mathrm{mM} \mathrm{Ca}^{2+}$

$4.0 \mathrm{mM} \mathrm{Mg}{ }^{+}$

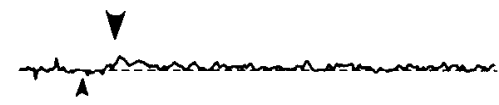

Figure 3. Separation of pre- and postsynaptic components of the optical signal. Left column shows extracellular field potentials, right column simultaneous optical recordings. Middle row shows electrical and optical signals associated with the afferent fibers (downward arrows) during block of synaptic transmission in a low-calcium Ringer's. Bottom row shows recovery of responses after 20 min wash in normal Ringer's. The records are the average of 16 responses. The trace durations are all 51.2 msec. Calibration on electrical records is $1 \mathrm{mV}$ by $2 \mathrm{msec}$, negative up.

for a distributed synaptic input is the deep retinal afferents of SGC and SAC described by Schmidt (1979).

\section{Postsynaptic component of optical signal}

Figure 3 shows simultaneously recorded extracellular field potentials and optical signals, before (top row), during (middle row), and after (bottom row) recovery from block of retinotectal synaptic transmission by superfusion of the slice wth $0.2 \mathrm{~mm}$ $\mathrm{Ca}^{2+}-4 \mathrm{mM} \mathrm{Mg}^{2+}$ Ringer's. During the block, only presynaptic potentials remain in the electrical recordings (indicated by downward arrow in Fig. 3, middle left trace). It is apparent (Fig. 3, middle right trace) that the major part of the optical
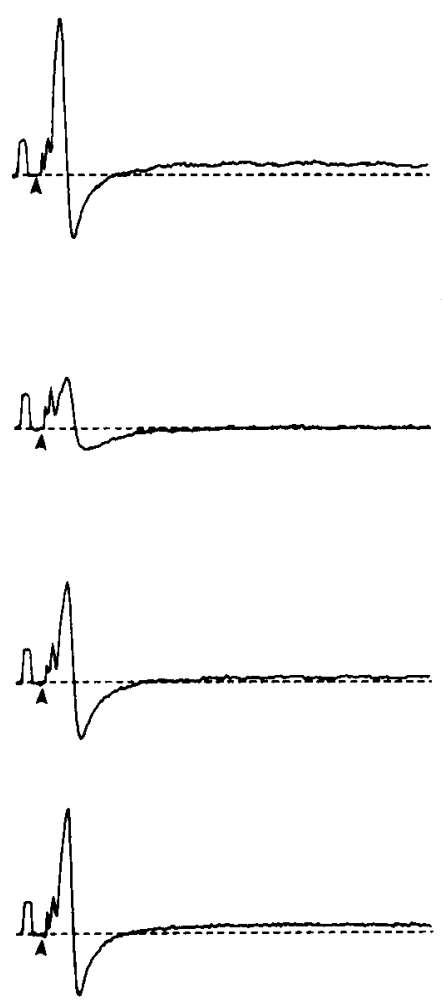
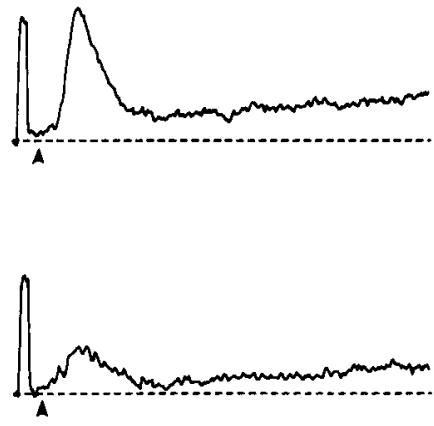

100

msec

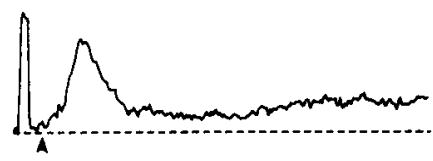

500

msec
Figure 4. Paired-shock depression of electrical and optical responses. Paired shocks were delivered to the optic nerve at the intervals shown to the right of the data. Field potentials are shown in the left column, simultaneously recorded optical responses are shown in the right column. Data shown are averages of 8 responses to the second shock of each pair (except for the top row labeled Control, which are the averaged responses to a single stimulus). Note particularly the simultaneous depression of the N1-N2 field potential and the Dl optical response at the 100 and 500 msec stimulus intervals. Baseline on optical records has been set to the fluorescence level immediately preceding the second stimulus of each pair. Thus, changes in resting fluorescence from the conditioning stimulus are not shown. Traces are $102 \mathrm{msec}$ in duration. Optical calibration is $5 \times 10^{-3} \Delta F / F, 2$ $\mathrm{msec}$ in duration. 
Figure 5. IPSP revealed by depolarizing the slice in $9 \mathrm{~mm} \mathrm{~K}^{+}$media. $A$ and $B$, Field potential and simultaneous optical recordings in SFGS observed in normal Ringer's ( $\left.3 \mathrm{~mm} \mathrm{~K}^{+}\right)$. $C$, Shows the enhancement of the $P 1$ wave (arrow), while the IPSP is revealed in $D$ (arrow) when the slice is bathed in 9 mM $\mathrm{K}^{+}$Ringer's. $E$ and $F$, Optical recordings in another slice. $E$ shows the response in normal Ringer's; $F$, the response in $9 \mathrm{~mm} \mathrm{~K}^{+}$Ringer's. Note the long-duration IPSP following the initial EPSP in $F$. Traces $A-D$ are $51.2 \mathrm{msec}$ duration, $E$ and $F$ are $102 \mathrm{msec}$ duration. Optical calibration pulses are $5 \times$ $10^{-3} \Delta F / F, 2$ msec duration.
$A$

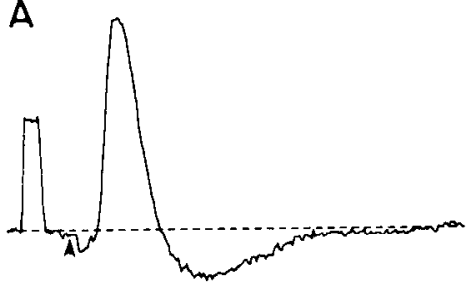

C

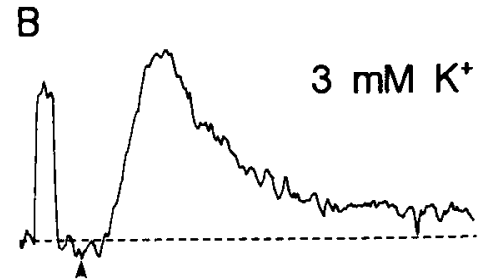

$\mathrm{D}$

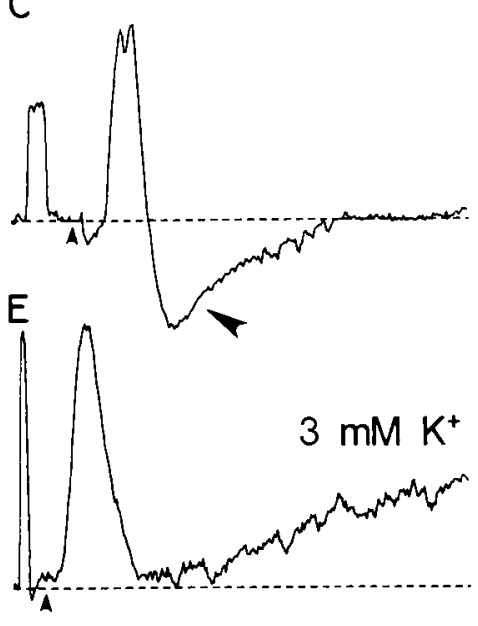

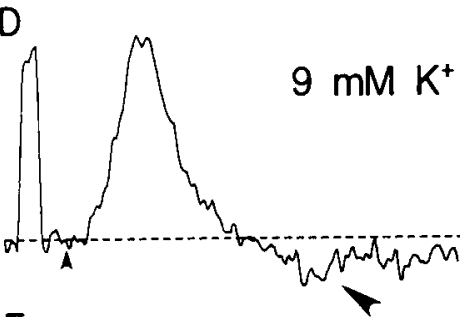

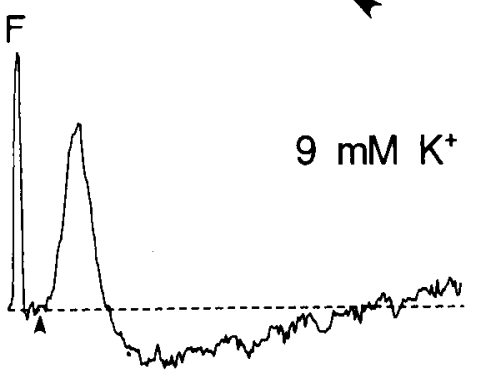

response is associated with postsynaptic cvents. A very small, short-latency optical response sometimes remains (downward arrow, middle right trace; see also Fig. 8). This event, as will be shown later, is associated with activity in the afferent (and/or efferent) fibers and their ensheathing glial cells.

The presumed monosynaptic wave of the extracellular field potential (N2, N3) undergoes a long-lasting depression when tested in a paired-pulse paradigm using supramaximal stimuli in normal Ringer's (left column, Fig. 4; see also Vanegas et al., 1971; Schmidt, 1979; Teyler et al., 1981). The short-latency optical signal (D1) undergoes a parallel depression (right column, Fig. 4). Thus, it is likely that the short latency D1 optical signal reflects the monosynaptic and/or disynaptic EPSPs in the dendrites of tectal neurons. The amplitude of the late component (D3) covaries with the D1 signal, although it appears to be reduced proportionately less than the $\mathrm{D} 1$.

\section{IPSP revealed in high $K^{+}$media}

One of the difficulties that optical recordings shares with all extracellular physiological approaches is the inability to control the membrane potential of the cells of interest. However, a qualitative adjustment of membrane potential may be made by altering the extracellular potassium concentration. We used this approach to depolarize cells in the slice by increasing the extracellular potassium in the bathing solution.

In some slices, we observed an increase in the fluorescence above resting following the D1 when the extracellular potassium level was raised. The top 2 rows of Figure 5 compare extracellular field potentials $(A, C)$ and optical responses $(B, D)$ as recorded in normal $3 \mathrm{mM} \mathrm{K}^{+}(A, B)$ and in $9 \mathrm{~mm} \mathrm{~K}^{+}(C, D)$. (Assuming a membrane with perfect potassium selectivity, an intracellular $\mathrm{K}^{+}$of $140 \mathrm{~mm}$, and using the bathing Ringer's for extracellular ion activities, the cells would be depolarized by about $22 \mathrm{mV}$ in the $9 \mathrm{mM} \mathrm{K}^{+}$media relative to the $3 \mathrm{mM} \mathrm{K} \mathrm{K}^{+}$ media. The actual depolarization is probably somewhat less than this because the membrane is not perfectly selective for potassium.) In the field potential recordings, the positive wave (P1) following the presumed monosynaptic current sink is enhanced in the high extracellular potassium solution. This enhancement is associated with a change in the time course of the P1 field potential. Under these conditions, a clear net hyperpolarization appears in the optical recordings (arrow, Fig. $5 D$ ). The bottom row shows optical recordings in another slice on a longer time scale. Recordings in normal (Fig. $5 E$ ) and $9 \mathrm{~mm}$ (Fig. $5 F$ ) potassium again illustrate the appearance of the hyperpolarization in the higher extracellular potassium superfusate. The fact that a net hyperpolarization is observed from the entire population of cells in the field of view suggests that the hyperpolarization of individual cells must be relatively large or that the hyperpolarization must be occurring in most of the tectal cells. Although the IPSP is evident only several milliseconds after the extracellular P1 potential, it appears to begin during the falling phase of the early EPSP (D1), simultaneously with the P1 potential. Laminar mapping experiments showed that the IPSP was maximal in SFGS and SGC.

In order to investigate whether the hyperpolarization might be an IPSP mediated by GABA, we examined its sensitivity to bath application of 2-10 $\mu \mathrm{M}$ bicuculline methiodide. Figure 6 shows the effects of $10 \mu \mathrm{M}$ bicuculline methiodide on the electrical and optical signals in normal media ( $3 \mathrm{mM} \mathrm{K}^{+}$). (We were unable to successfully test the effects of even low concentrations of bicuculline in high $\mathrm{K}^{+}$media. In high $\mathrm{K}^{+}$media, bicuculline induced a spreading depression of evoked activity, rendering recordings of synaptic events impossible.) Each row of records illustrates, from left to right, the extracellular field potential, a short-duration ( $50 \mathrm{msec}$ ) optical recording, and a long-duration 
Field Potentials
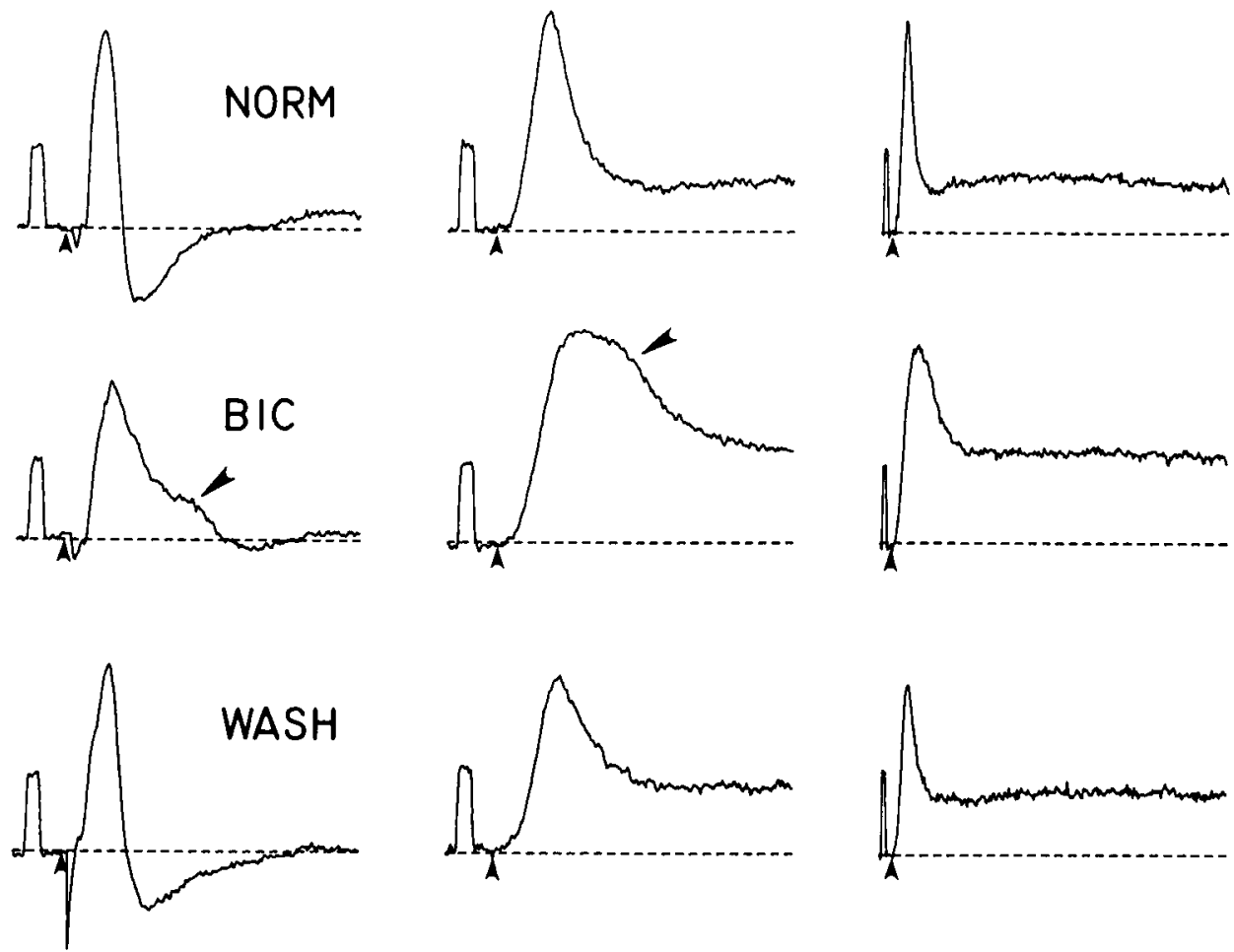

Figure 6. Effects of bath-applied 10 $\mu \mathrm{M}$ bicuculline methiodide on electrical and optical responses in SFGS. The top row (NORM) shows records obtained in normal Ringer's. Left and middle columns are the extracellular field potential and simultaneously recorded optical responses (with $51.2 \mathrm{msec}$ trace duration). Right column shows the optical response with a 200 -msec-long trace to show the D3 potential more clearly. Middle row (BIC) shows the effect of incubating the slice in $10 \mu \mathrm{M}$ bicuculline methiodide for $20 \mathrm{~min}$. Note the loss of the P1 in the field potential records (arrow) and the apparent second EPSP (or lengthening of the D1 response) in the optical signal (arrow). Also note the increase in the magnitude of the D3 optical signal in the right column. Bottom row (WASH) shows the recovery of the extracellular field potentials and optical responses after 40 min wash in normal Ringer's. Electrical calibration pulse $1 \mathrm{mV}$ by 2 msec. Optical calibration pulse $5 \times 10^{-3}$ $\Delta F / F, 2 \mathrm{msec}$ in duration.
(200 msec) optical recording. The top row shows responses in normal Ringer's. Bath application of $10 \mu \mathrm{M}$ bicuculline (middle row) abolishes (or reverses) the P1 wave of the field potential and produces an increase in the latency and duration of the optically recorded D1 depolarization: The increase in duration of the initial EPSP appears to be associated with the presence of a second (presumably disinhibited) EPSP occurring during the falling phase of the D1 EPSP (arrow). Although it is not evident from these averaged records, the depolarization during the falling phase of the D1 seemed to consist of a slow event of somewhat variable latency. Sometimes the Dl and second EPSP could be distinguished by an inflection towards baseline occurring between them. The late (D3) potential is also enhanced in the presence of bicuculline. The lower row shows the return to control after the bicuculline has been washed out for $40 \mathrm{~min}$.

In slices bathed in $2 \mu \mathrm{M}$ bicuculline, the P1 field potential was reduced in amplitude without producing evidence of the second EPSP. A smaller increase in the duration of the D1 component and in the amplitude of the D3 component was observed. Bath application of $50 \mu \mathrm{M}$ strychnine or $100 \mu \mathrm{M} d$-tubocurarine produced changes in the extracellular field potentials and optical responses similar to those seen with $10 \mu \mathrm{M}$ bicuculline (not shown).

\section{Possible glial origin of late optical signal}

The late optical signal (D3) had a long duration and a dependence on earlier synaptic events that suggested that it might be glial in origin. If the D3 signal is glial in origin, we would expect it to vary in amplitude with changes in resting membrane potential (produced by changes in the extracellular potassium concentration) and to summate with repetitive stimulation.

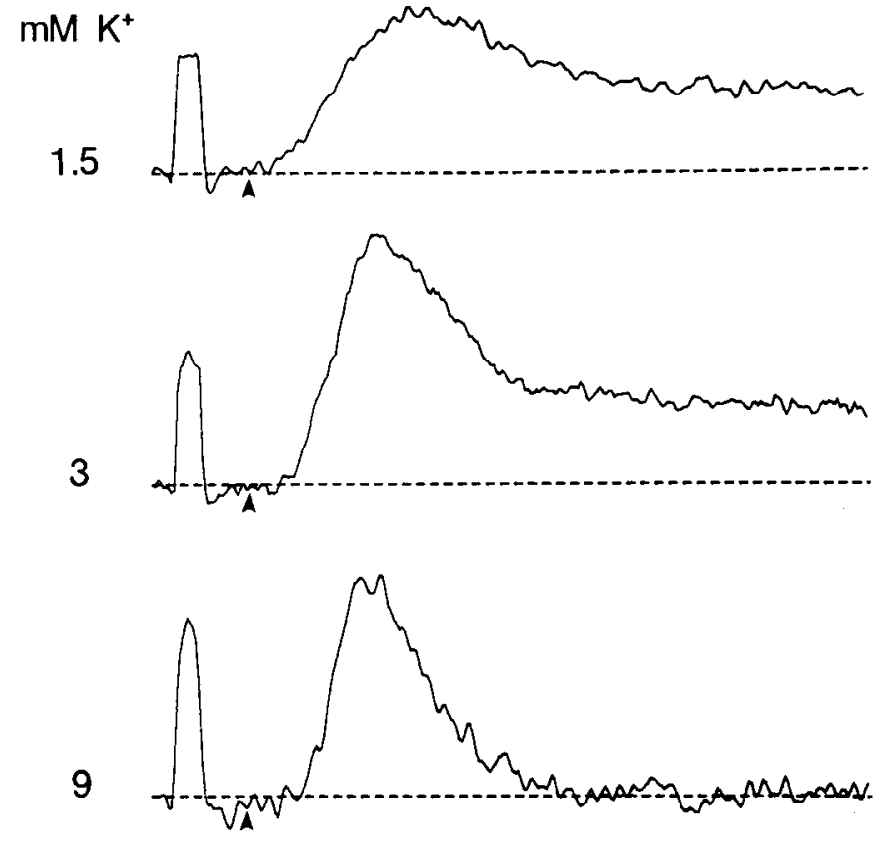

Figure 7. Effects of varying extracellular $\mathrm{K}^{+}$on optical signals in SFGS. The bath $\mathrm{K}^{+}$level is shown to the left of the traces. Note that as the $\mathrm{K}^{+}$ level is increased, the D3 signal becomes smaller and, in this experiment, was virtually absent in $9 \mathrm{~mm} \mathrm{~K} \mathrm{~K}^{+}$. Note also the changes in the configuration of the D1 potential. The $9 \mathrm{mM} \mathrm{K}^{+}$trace has been smoothed with a 3 point formula to decrease some high-frequency noise in the recording. Optical calibrations are $5 \times 10^{-3} \Delta F / F, 2 \mathrm{msec}$ in duration. 

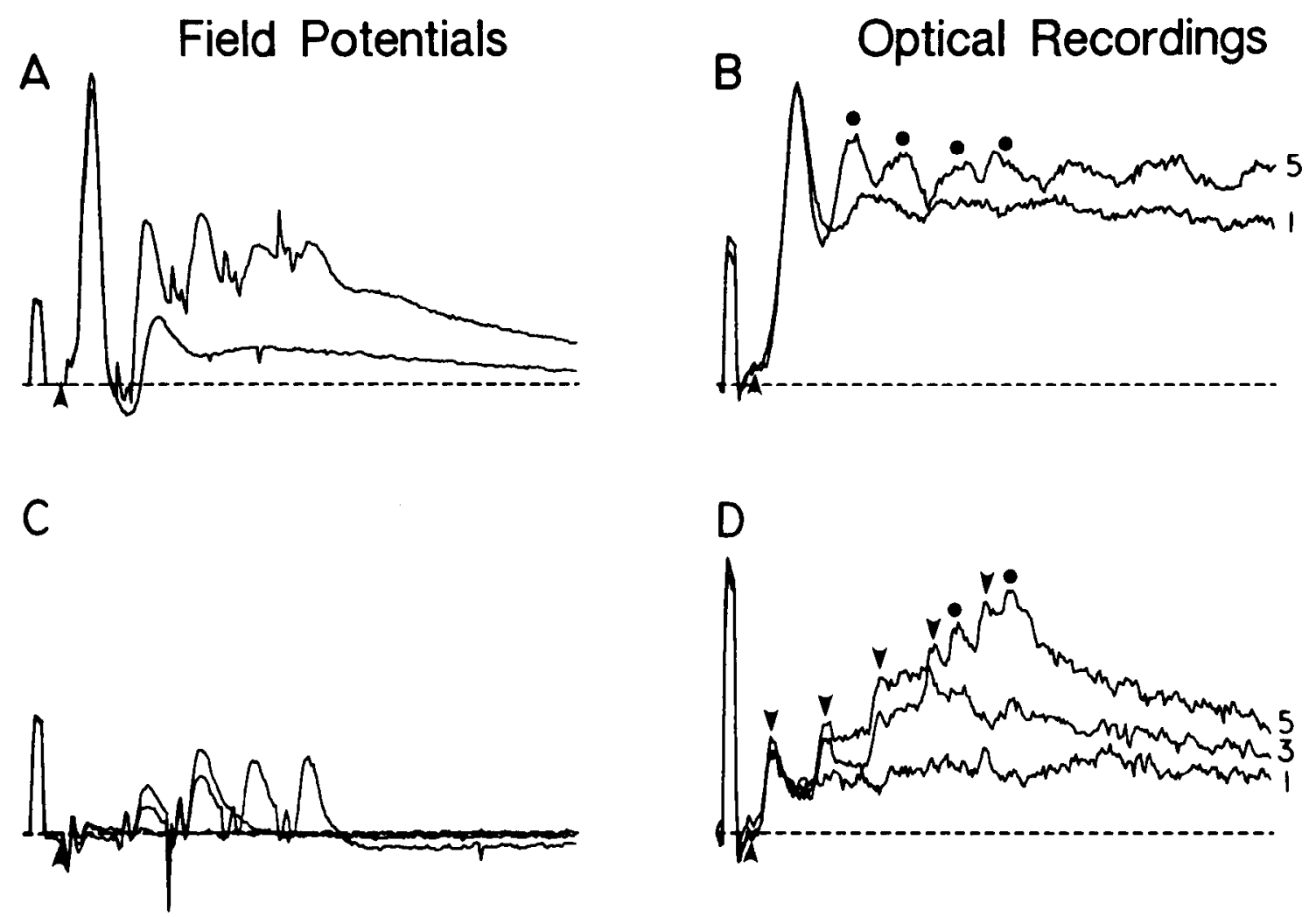

Figure 8. Effects of repetitive stimulation on the slow optical signal. The top panels of this figure $(A, B)$ illustrate that a slight increase in the magnitude of the slow D3 event can be observed when the optic nerve is stimulated with a train of 5 stimuli at $100 \mathrm{~Hz}$ relative to the response to a single shock. In the lower panels $(C, D)$, synaptic transmission was blocked with a medium containing $0.1 \mathrm{~mm} \mathrm{Ca}^{2+}$ and $4.0 \mathrm{~mm} \mathrm{Mg}^{2+}$. With single stimuli, there was no postsynaptic response. When trains of stimuli were presented, a small postsynaptic event appeared in the field potential records $(C)$. The optical signals $(D)$ showed a small but well-defined fast event associated with the afferent fiber volley (arrows), followed by a longlasting depolarization. Although the developing EPSP makes interpretation difficult, the slow event appeared to summate with repeated stimuli in a monotonic fashion. Small postsynaptic potentials were also evident in the optical recordings (small dots above traces). All optical calibrations are $2 \times 10^{-3} \Delta F / F, 2 \mathrm{msec}$ in duration.

We qualitatively investigated the dependence of the $\mathrm{D} 3$ signal on membrane potential by varying extracellular potassium concentration. There is a monotonic decrease in the relative amplitude of the D3 signal as the extracellular $\mathrm{K}^{+}$is increased from 3 to $9 \mathrm{~mm}$ and a slight increase when the extracellular $\mathrm{K}^{+}$was decreased to $1.5 \mathrm{~mm}$ (Fig. 7). The effects on the late optical signal (D3) were more pronounced than on the earlier signals (D1, D2). Similar results were obtained in 3 separate experiments. Attempts to record with higher extracellular $\mathrm{K}^{+}$levels were unsuccessful because these concentrations caused spreading depression in the slice. These experiments are not readily amenable to quantitative analysis because the amplitude of the D3 optical signal depends on D1, and the D1 optical signal (and the N2-N3 field potential amplitude) was clearly affected by the changing extracellular $\mathrm{K}^{+}$levels. (The changes in the D1 could be produced by potential-dependent alterations in transmitter release from the presynaptic terminals or by changes in synaptically activated voltage-dependent membrane conductances in the postsynaptic cells.) Nonetheless, these data show that the late optical signal (D3) varies in a fashion that is consistent with a glial origin. Other possibilities are considered in the Discussion.

Figure $8 A$ shows that with repetitive stimulation of the optic tract, the field potentials in the tectal slice exhibit substantial depression. The intervening synaptic mechanisms make interpretation of a summation cxperiment difficult. Nonetheless, the optical signals (Fig. $8 B$ ) show an increase in the magnitude of the late signal with 5 stimuli at $100 \mathrm{~Hz}$ over the response to a single stimulus. When we blocked synaptic transmission (Fig. $8 C$ ), the optical signal in response to a single shock showed a fast depolarization (arrows, Fig. $8 D$ ) followed by a long depolarization. It appeared that the slow depolarization might be glial also. When repetitive stimuli were delivered to the optic tract, a small EPSP developed. However, the optical recordings revealed a regular increment in the amplitude of the slow depolarization with increasing numbers of shocks in the train. (This summation is not evident in Fig. 4 because the recording baseline level was reset to zero before each response. Thus, any residual depolarization resulting from the first stimulus of the pair could not be measured in that experiment.) This slow depolarization exhibited a similar summation in experiments where the EPSP was completely blocked. Thus, this signal probably represents depolarization of glial cells surrounding the afferent optic nerve axons.

\section{Discussion}

We have shown that fluorescent voltage-sensitive dyes can be used to study electrical events in goldfish tectal slices maintained in vitro. In spite of their apparent simplicity, the optical signals consist of several different components that can be correlated with known tectal physiology. The earliest event appears to be associated with action potentials in the afferent fibers and is 
followed by the depolarization of surrounding glial cells. The largest event (D1) is probably the monosynaptic/disynaptic FPSP of postsynaptic dendritic elements. This is followed by an inhibitory postsynaptic potential (D2), which is best demonstrated in slices depolarized by elevating the extracellular potassium concentration. Another component is a long-lasting depolarization (D3) whose magnitude varies with the magnitude of early postsynaptic events and external potassium concentration and that shows summation with repetitive stimulation. This event probably has a glial origin. In addition, we observed an EPSP following the monosynaptic EPSP that arose following application of convulsant drugs, probably as a consequence of disinhibition. We also observed a small depolarization occurring simultaneously with the $\mathrm{N} 4$ wave in slices incubated with low concentrations of dye. We will discuss briefly some general considerations of fluorescence rccordings in tissuc slices, followed by the interpretation of our main observations in the goldfish tectal slice.

\section{Factors affecting the magnitude of the optical signal in slices}

Several factors can affect the relative magnitude of the optical signal in slice preparations, making a cautious interpretation of the amplitudes of dye responses necessary. Some of these limitations have been discussed previously (Grinvald et al., 1982b; Orbach and Cohen, 1983; Grinvald, 1985). One limitation that is unique to fluorescence recordings in brain slices is the amount of damaged tissue in the slice. Ultrastructural examination of physiologically viable brain slices from several preparations has revealed that cells in the outer $50-75 \mu \mathrm{m}$ adjacent to the cut surfaces are often swollen and pyknotic (Yamamoto et al., 1970; Jahnsen and Laursen, 1983). As has been noted previously, dead cells fluoresce more brightly than live cells when stained with these dyes (Grinvald et al., 1982a). Since the fluorescence from dead or electrically inactive tissue does not contribute to the signal but will disproportionately increase the total resting fluorescence, the signal-to-noise ratio is degraded compared to that potentially attainable in the absence of damaged tissue. As the fluorescence signal from the damaged tissue is spatially localized to the cut surfaces of the slice, it may be possible to reduce the contribution of the damaged tissue by using optical sectioning techniques (such as confocal microscopy). Because we have not used such techniques, it is likely that the actual fractional fluorescence change occurring in the viable cells of the middle of the slice is somewhat larger than we have recorded. A previous study using optical recording in slices (Grinvald et al., 1982b) used absorption dyes. The light scattering signals were nearly' as large as the absorption signals and had to be subtracted from the absorption signal to isolate the potential-dependent components. Because the signals from fluorescent dyes are larger by 1-2 orders of magnitude than the scattering signals, the scattering signals are much less of a problem with fluorescence measurements. Grinvald (1985) has indicated that absorbance measurements should, in principle, produce better signal-to-noise ratios than fluorescence measurements in multilayer preparations such as slices. However, our best $\mathrm{S} / \mathrm{N}$ ratios are comparable to those obtained with absorption dyes in hippocampal slices, and we have also obtained fluorescence signals from rat hippocampal slices with equivalent $\mathrm{S} / \mathrm{N}$ ratios. We have been able to obtain better $\mathrm{S} / \mathrm{N}$ ratios using $40 \times 0.75 \mathrm{NA}$ and $63 \times 1.25$ NA objectives with a slightly larger illuminated area (i.e., Fig. $8 D$ ). Attendant to these improvements, however, is an increase in bleaching and photodynamic damage.

\section{Early optical response (DI)}

Our observations indicate that the Dl optical signal represents a mono- or disynaptic EPSP in the dendrites of tectal neurons innervated by the optic nerve. Matsumoto et al. (1983) and Vanegas et al. (1974) showed, using intracellular recording, that monosynaptic EPSPs could be obtained from neurons in SFGS and SGC. The duration, latency, and overall shape of these EPSPs closely parallel the D1 optical signal. However, our recordings probably include contributions from other cell populations (see below). Matsumoto et al. (1983) also reported EPSPs that had longer latencies indicative of polysynaptic activation. Because the dye signal represents the superposition of membrane potentials in a heterogenous population of cells, we cannot unequivocally determine if, or to what extent, the D1 signal may reflect polysynaptic versus monosynaptic EPSPs.

The spatial distribution of the early optical response indicates that it arises from neurons whose processes extend from, or are encompassed within, SO to SPV, but not SM. Any and all neurons that receive optic nerve synapses and whose cell processes are confined to these layers may contribute significantly to this response. Because it appeared that no signal associated with D1 was present in SM (the SM signal appears to reflect the start of the D3 event), Meek's (1983) type I and II neurons probably do not contribute significantly to the optical signal. This is consistent with the relatively sparse innervation of these cells by the optic nerve (Meek, 1983, Appendix I). The sheer number of type XIV neurons relative to all other types would suggest that the optical signal may be dominated by postsynaptic potentials in these cells. The cell bodies of these neurons lie primarily in SPV. It is not surprising that the optical signal in SPV is small, as the total surface area of the nearly spherical cell bodies in SPV is less than that of their dendrites. A similar decrease in signal size at the cell body layer was noted for the stratum pyramidale of the rat hippocampus (Grinvald et al., $1982 \mathrm{~b})$. It is possible that the somatic membrane stains less or the dye is less responsive to membrane potential when in the somatic membrane than in the dendritic membrane. Also, there are undifferentiated cells in SPV (Meyer, 1978; Stcvenson and Yoon, 1978), which would contribute to the resting fluorescence but would be unresponsive to optic tract stimulation, thereby reducing the apparent signal size. The recordings from SPV may have actually included a smaller volume of tissue because the edge of the slice may have been tilted relative to the horizontal plane (note that the fluorescence calibration pulse is smaller, indicating less total fluorescence was present).

Although the type XIV cells are likely to be a major contributor to the optical signals, other cell types in the tectum should also make important contributions to the total optical signal. With extracellular staining methods, it will be very difficult to isolate the contributions of different cell types to the optically recorded population response.

\section{Inhibitory potentials (D2)}

The existence of inhibitory events in the optic tectum of lower vertebrates following optic nerve stimulation has been well documented (Sutterlin and Prosser, 1970; Vanegas et al., 1971, 1974; O'Benar, 1976; Sajovic and Levinthal, 1983; Freeman and Norden, 1984; Grinvald et al., 1984). In goldfish tectal neurons, IPSPs have been noted by Matsumoto et al. (1983) and Freeman and Norden (1984) using intracellular recording. Evidence for inhibitory inputs to tectal cells following optic 
nerve stimulation has also been obtained in Eugerres by Vanegas et al. (1974) and in zebra fish by Sajovic and Levinthal (1983). An inhibitory input at the level of SFGS was suggested by Sajovic and Levinthal (1983), from pharmacological evidence and current source-density analysis. Appropriate intrinsic neurons in the tectum that appear to use the inhibitory amino acid transmitter GABA have also been identified (Villani et al., 1981).

The spatial distribution of the IPSP seen with the voltagesensitive dyes suggests that the IPSP is maximal in SFGS, in agreement with Sajovic and Levinthal (1983). It is interesting to note that elevation of extracellular potassium, which helped reveal the IPSP, also consistently enhanced the $P 1$ field potential as recorded in SFGS (Fig. 5). If the Pl field potential corresponded to an EPSP generated deeper in the tectum, then depolarization of the tectal cells by raised extracellular potassium would have bcen expected to depress it rather than increase it. Similarly, we would not expect bicuculline to cause the positive wave to "reverse" in SFGS if it were the passive component of a remote excitatory current sink (see Fig. 5). The latency of the optically recorded IPSP is difficult to determine because the IPSP is superimposed on the D1 and D3 depolarizations. When the records obtained in the presence of $2 \mu \mathrm{M}$ bicuculline are subtracted from the normal controls, it is found that the bicuculline-sensitive portion of the response actually begins during the falling phase of the DI EPSP. This is consistent with changes observed in field potential recordings, in which the monosynaptic EPSP has been isolated by using low- $\mathrm{Ca}^{2+}$ bathing solutions (Langdon et al., 1987). The falling phase of the N2-N3 potential in low- $\mathrm{Ca}^{2+}$ is much slower than in normal Ringer's, suggesting that it is normally terminated by a current of opposite polarity. Thus, there appears to be an inhibitory conductance occurring in cells and their processes in SFGS and SGC that begins during the falling phase of the optic EPSP. The extracellularly recorded Pl field potential appears to be correlated with this IPSP, both because of its enhancement in a depolarizing media and its comparable onset latency.

Experiments with bath application of bicuculline methiodide, a GABA-receptor antagonist, are also consistent with the observations of Sajovic and Levinthal (1983). However, we have also observed a similar change in both the extracellular field potentials and population intracellular optical responses with bath application of strychnine $(50 \mu \mathrm{M})$ and $d$-tubocurarine (10$100 \mu \mathrm{M}$ ) (see also Langdon and Freeman, 1987). Both Buser (1951) and Konishi (1960) observed an alteration in the configuration of the extracellular field potentials similar to that seen here with topical application of strychnine to the goldfish tectum in vivo. While it would tempting to conclude that the IPSP is indeed generated through local GABA-aminergic circuits, further pharmacological characterization of the IPSP is necessary. For example, direct membrane effects of bicuculline (when used above $7 \mu \mathrm{M}$ ) have been reported in cultured neonatal rat spinal cord neurons (Heyer et al., 1982). Similarly, $10 \mu \mathrm{M} d$-tubocurarine has recently been shown to have antagonistic effects at GABA receptors in the hippocampus (Lebeda et al., 1982). The ovcrlapping and somctimcs nonspccific actions of these agents need to be investigated in more detail using iontophoretic application of the putative transmitters and their antagonists before firm conclusions about the pharmacology of local inhibitory tectal events can be drawn.

Schmidt interpreted the "P3" (his terminology, corresponding in time to our P1) current source as the passive component of excitatory synaptic input at the level of SAC to radial cells (e.g., a deep excitatory afferent input), and demonstrated using anterograde cobalt labeling that some optic nerve afferents terminated in a narrow band in SAC. Because depolarization of the slice with $\mathrm{K}^{+}$resulted in a complex time course of the P1 wave, our results are not entirely incompatible with the existence of a simultaneous excitatory input to the deeper tectal layers. The time course of synaptic activation may be slightly different in the in vitro versus the in vivo situations because of the different sites of optic nerve stimulation. Thus, the synaptic currents produced by deeper afferents may occur earlier in vitro than in vivo so that they are superimposed on part of the N2-N3 field potentials and on the D1 optical signal. If the latency of these afferents were short enough in vitro, they could account for the relatively constant rise time of the D1 potential in the deeper layers. Alternatively, the deeper excitatory inputs may be weak and partially masked by the synergistic current flows produced by the more superficially generated IPSP and P1 wave that we have identified.

\section{Late optical response (D3)}

The late optical response, or D3 signal, appears to be graded with the magnitude of the D1 signal but has a short latency and long duration. Further, the D3 signal is seen in all tectal layers following optic tract stimulation. One set of cells that might produce such a spatially distributed signal are the radial glial cells (Stevenson and Yoon, 1982). The glial cell bodies reside in SPV, and their processes extend from the ventricular surface to the pial surface of the tectum. The processes of the glial cells are sparse in SO but exhibit uniform branching in all other layers. The late optical signal was slightly smaller in SO, possibly due to the lower membrane area of radial glial cells in that layer (Stevenson and Yoon, 1982). Further, the dependence of this signal on extracellular potassium is consistent with it being at least a depolarizing response with a reversal or null potential well below that of the early EPSP. Our evidence for summation during repetitive stimulation of the optic tract, particularly in the absence of significant synaptic transmission, also supports the contention that at least part of this event is glial in origin. Our conclusion is also supported by the recent report of LevRam and Grinvald (1986). They identified and characterized an optical signal of glial origin in the rat optic nerve. They also noted that the dye RH414 had a greater affinity for binding to glial cell membranes than to neural cells in culture, which would tend to increase the detected glial potential changes relative to the neural potential changes. Optical and intracellular recordings of glial potentials in frog optic nerve have been compared by Konnerth and Orkand (1986). These recordings confirmed the similar time courses of the slow optical signals and the glial depolarizations during repetitive stimulation.

It is also conceivable that the D3 is partially generated by a slow synaptically mediated EPSP. The changes in amplitude with extracellular $\mathrm{K}^{+}$could then result from changes in a simultaneously occurring inhibitory potential or from changes in synaptic transmission through polysynaptic pathways. Extraccllular potassium concentration measurements and intracellular recordings from identified glial cells will be necessary to confirm the origin of this optical signal.

\section{Summary}

We have examined the optical signal produced by fluorescent voltage-sensitive dyes in the goldfish optic tectum slice in vitro and have provided evidence for the origins of some of the major 
optical events. The optical recordings have helped identify an initial EPSP, followed by an IPSP and a slow (likely glial) depolarization. Additional activity in the optic nerve afferent fibers, a disinhibited EPSP in the presence of bicuculline, and a small EPSP associated with a late (N4) potential were also identified. The optical recording technique employed in conjunction with field potential recording appears to be a powerful method for the analysis of local synaptic circuits on a population level. The recordings primarily represent postsynaptic activity in small neurons, which previously have proved recalcitrant to intracellular recordings. One of the advantages of optical recording is the ability to record over a large region of neural tissue simultaneously using a photodiode array (Grinvald et al., 1981, 1984). Thus, it should be possible to perform spatial mapping of postsynaptic events in the goldfish tectum relatively rapidly, as has already been done in the frog tectum (Grinvald et al., 1984). Some preliminary experiments of this type have been reported in goldfish by Anglister and Grinvald (1984). The analysis of the optical signal presented here should aid significantly in the interpretation of optical signals in such a mapping study.

\section{References}

Anglister, L., and A. Grinvald (1984) Real time visualization of the spatio-temporal spread of electrical responses in the optic tectum of vertebrates. Isr. J. Med. Sci. 20: 458.

Attardi, D. G., and R. W. Sperry (1963) Preferential selection of central pathways by regenerating optic fibers. Exp. Neurol. 7: 46-64.

Boss, V. C., and J. T. Schmidt (1984) Activity and the formation of ocular dominance patches in dually innervated tectum of goldfish. $\mathbf{J}$. Neurosci. 4: 2891-2905.

Buser, P. (1951) Modifications, par la strychnine, de la response du lobe optique de poisson. Essai d'interpretation. J. Physiol. (Paris) 43: 673-677.

Chung, S. H., M. J. Keating, and T. V. P. Bliss (1974) Functional synaptic relations during the development of the retino-tectal projection in amphibians. Proc. R. Soc. London [Biol.] 187: 449-459.

Cohen, L. B., and B. M. Salzberg (1978) Optical measurement of membrane potential. Rev. Physiol. Biochem. Pharmacol. 83: 35-88.

Cohen, L. B., R. D. Keynes, and D. Landowne (1972) Changes in axon light-scattering that accompany the action potential: Current dependent components. J. Physiol. (Lond.) 224: 727-752.

Cohen, L. B., B. M. Salzberg, H. V. Davila, W. N. Ross, D. Landowne, A. S. Waggoner, and C. H. Wang (1974) Changes in axon fluorescence during activity: Molecular probes of membrane potential. $\mathrm{J}$. Membr. Biol. 19: 1-36.

Cook, J. E., E. C. C. Rankin, and H. P. Stevens (1983) A pattern of optic axons in the normal goldfish tectum consistent with the caudal migration of optic terminals during development. Exp. Brain Res. 52: 147-151.

Freeman, J. A. (1971) An electronic stimulus artifact suppressor. Electroencephalogr. Clin. Neurophysiol. 31: 170-172.

Freeman, J. A. (1977) Possible regulatory function of acetylcholine receptor in maintenance of retinotectal synapses. Nature 269: 218222.

Freeman, J. A. (1979a) Intracellular responses and receptor localization of neurons in slices of goldfish tectum. Invest. Ophthalmol. (Suppl. 5) 18: 228 .

Freeman, J. A. (1979b) Dendritic localization and density of acetylcholine receptors in single cells in slices of goldfish optic tectum. Soc. Neurosci. Abstr. 5: 740 .

Freeman, J. A., and J. J. Norden (1984) Neurotransmitters in the optic tectum of nonmammalians. In Comparative Neurology of the Optic Tectum, H. Vanegas, ed., pp. 469-546, Plenum, New York.

Grinvald, A. (1985) Real-time optical mapping of neuronal activity: From single growth cones to the intact mammalian brain. Annu. Rev. Neurosci. 8: 263-305.

Grinvald, A., and M. Segal (1984) Optical monitoring of electrical activity. In Brain Slices, R. Dingledine, ed., pp. 227-261, Plenum, New York.
Grinvald, A., L. B. Cohen, S. Lesher, and M. B. Boyle (1981) Simultaneous optical monitoring of activity of many neurons in invertebrate ganglia using a 124-element photodiode array. J. Neurophysiol. 45: $829-840$.

Grinvald, A., R. Hildesheim, I. C. Farber, and L. Anglister (1982a) Improved fluorescent probes for the measurement of rapid changes in membrane potential. Biophys. J. 39: 301-308.

Grinvald, A., A. Manker, and M. Segal (1982b) Visualization of the spread of electrical activity in rat hippocampal slices by voltagesensitive optical probes. J. Physiol. (Lond.) 333: 269-291.

Grinvald, A., A. Fine, I. C. Farber, and R. Hildesheim (1983) Fluorescence monitoring of electrical responses from small neurons and their processes. Biophys. J. 42: 195-198.

Grinvald, A., L. Anglister, J. A. Freeman, R. Hildesheim, and M. Manker (1984) Real-time optical imaging of naturally evoked electrical activity in intact frog brain. Nature 308: 848-850.

Gupta, L. K., B. M. Salzberg, A. Grinvald, L. B. Cohen, K. Kamino, S. Lesher, M. B. Boyle, A. S. Waggoner, and C. H. Wang (1981) Improvements in optical methods for measuring rapid changes in membrane potential. J. Membr. Biol. 58: 123-127.

Heyer, E. J., L. M. Nowak, and R. L. Macdonald (1982) Bicuculline: A convulsant with synaptic and non-synaptic actions. Neurology 31 : 1381-1390.

Jahnsen, H., and A. M. Laursen (1983) Brain slices. In Current Methods in Cellular Neurobiology, vol. 3, J. L. Barker and J. F. McKelvy, eds., pp. 189-224, Wiley, New York.

Konishi, J. (1960) Electric response of visual center to optic nerve stimulation in fish. Jpn. J. Physiol. 10: 28-41.

Konnerth, A., and R. K. Orkand (1986) Voltage-sensitive dyes measure potential changes in axons and glia of the frog optic nerve. Neurosci. Lett. 66: 49-54.

Langdon, R. B., and J. A. Freeman (1987) Pharmacology of retinotectal transmission in the goldfish: Effects of nicotinic ligands, strychnine, and kynurenic acid. J. Neuroscience 7: 760-773.

Langdon, R. B., P. B. Manis, and J. A. Freeman (1987) Goldfish retinotectal transmission in vitro: Component current sink-source pairs isolated by varying calcium and magnesium levels. Brain Res. (in press).

Lebeda, F. J., J. J. Hablitz, and D. Johnston (1982) Antagonism of GABA-mediated responses by d-tubocurarine in hippocampal neurons. J. Neurophysiol. 48: 622-632.

Lev-Ram, V., and A. Grinvald (1986) $\mathrm{Ca}^{2+}$ - and $\mathrm{K}^{+}$-dependent communication between central nervous system myelinated axons and oligodendrocytes revealed by voltage-sensitive dyes. Proc. Natl. Acad. Sci. USA 83: 6651-6655.

Loew, L. M., L. B. Cohen, B. M. Salzberg, A. L. Obaid, and F. Bezanilla (1985) Charge-shift probes of membrane potential. Characterization of aminostyrylpyridinium dyes on the squid giant axon. Biophys. J. 47: 71-77.

Manis, P. B., and J. A. Freeman (1984) Voltage-sensitive dyes signal synaptic potentials in goldfish optic tectum in vitro. Soc. Neurosci. Abstr. 10: 1076.

Manis, P. B., and J. A. Freeman (1985) Optical recordings of neural activity in the goldfish optic tectum. Invest. Ophthalmol. (Suppl.) 26: 264.

Matsumoto, N., and T. Bando (1981) Long-lasting evoked potential and repetitive firing recorded from the carp optic tectum in $\mathrm{Cl}$-deficient medium in vitro. Brain Res. 225: 437-441.

Matsumoto, N., H. Kiyama, and T. Bando (1983) An intracellular study of the optic tectum of the carp in vitro. Neurosci. Lett. 38: 1722.

Meek, J. (1983) Functional anatomy of the tectum mesencephali of the goldfish. An explorative analysis of the functional implications of the laminar structural organization of the tectum. Brain Res. Rev. 6: 247-297.

Meyer, R. L. (1978) Evidence from thymidine labeling for continuing growth of retina and tectum in juvenile goldfish. Exp. Neurol. 59: 99-111.

Migani, P., A. Contestabile, G. Cristini, and V. Labanti (1980) Evidence of intrinsic cholinergic circuits in the optic tectum of teleosts. Brain Res. 194: 125-135.

O'Benar, J. D. (1976) Electrophysiology of neural units in the goldfish optic tectum. Brain Res. Bull. 1: 529-541.

Orbach, H. S., and L. B. Cohen (1983) Optical monitoring of activity from many areas of the in vitro and in vivo salamander olfactory bulb: 
A new method for studying functional organization in the vertebrate central nervous system. J. Neurosci. 3: 2251-2262.

Orbach, H. S., L. B. Cohen, and A. Grinvald (1985) Optical mapping of electrical activity in rat somatosensory and visual cortex. J. Neurosci. 5: 1886-1895.

Orkand, R. K., J. G. Nicholls, and S. W. Kuffler (1966) Effect of nerve impulses on the membrane potential of glial cells in the central nervous system of amphibia. J. Neurophysiol. 29: 788-806.

Piller, H. (1977) Microscope Photometry, Springer-Verlag, Berlin.

Ross, W. N., B. M. Salzberg, L. B. Cohen, A. Grinvald, H. V. Davila, A. S. Waggoner, and C. H. Wang (1977) Changes in absorption, fluorescence, dichroism, and birefringence in stained giant axons: Optical measurement of membrane potential. J. Membr. Biol. 33: 141-183.

Rusoff, A. C. (1984) Paths of axons in the visual system of perciform fish and implications of these paths for rules governing axonal growth. J. Neurosci. 4: 1414-1428.

Sajovic, P., and C. Levinthal (1983) Inhibitory mechanisms in zebrafish optic tectum: Visual response properties of tectal cells altered by picrotoxin and bicuculline. Brain Res. 271: 227-240.

Schmidt, J. T. (1979) The laminar organization of optic nerve fibers in the tectum of goldfish. Proc. R. Soc. London [Biol.] 205: 287-306.

Schmidt, J. T., D. L. Edwards, and C. Struemer (1983) The re-establishment of synaptic transmission by regenerating optic axons in goldfish: Timc coursc and effects of blocking activity by intraocular injection of tetrodotoxin. Brain Res. 269: 15-27.

Stevenson, J. A., and M. G. Yoon (1978) Regeneration of optic nerve fibers enhances cell proliferation in the goldfish optic tectum. Brain Res. 153: 345-351.

Stevenson, J. A., and M. G. Yoon (1982) Morphology of radial glia, ependymal cells and periventricular neurons in the optic tectum of goldfish (Carassius auratus). J. Comp. Neurol. 205: 128-138.

Stuermer, C. A. O. (1984) Rules for retinotectal terminal arborizations in the goldfish optic tectum: A whole-mount study. J. Comp. Neurol. 229: 214-232.

Sutterlin, A. M., and C. L. Prosser (1970) Electrical properties of goldfish optic tectum. J. Neurophysiol. 33: 36-45.

Teyler, T. J., D. Lewis, and V. E. Shashoua (1981) Neurophysiological and biochemical properties of the goldfish optic tectum maintained in vitro. Brain Res. Bull. 7: 45-56.

Vanegas, H., E. Essayag-Millan, and M. Laufer (1971) Response of the optic tectum to stimulation of the optic nerve in the teleost $E u$ gerres plumieri. Brain Res. 31: 107-118

Vanegas, H., J. Amat, and E. Essayag-Millan (1974) Postsynaptic phenomena in optic tectum neurons following optic nerve stimulation in fish. Brain Res. 77: 25-38.

Villani, L., A. Poli, A. Contestabile, P. Migani, G. Cristini, and R. Bissoli (1981) Effect of kainic acid on ultrastructure and gamma-aminobutyrate-related circuits in the optic tectum of the goldfish. Neuroscience 6: 1393-1403.

Yamamoto, C., I. J. Bak, and M. Kurokawa (1970) Ultrastructural changes associated with reversible and irreversiblc suppression of electrical activity in olfactory cortex slices. Exp. Brain Res. 11: 360 372. 